The Astrophysical Journal, 198:545-549, 1975 June 15

(C) 1975. The American Astronomical Society. All rights reserved. Printed in U.S.A.

\title{
THE INTERSTELLAR LINES OF THE FEIGE STARS
}

\author{
J. G. Cohen And D. A. Meloy \\ Kitt Peak National Observatory,* Tucson, Arizona \\ Received 1974 November 18; revised 1974 December 27
}

\begin{abstract}
New measurements of the equivalent widths and radial velocities of the interstellar lines of $\mathrm{Ca}$ II and $\mathrm{Na}$ I in the spectra of Feige stars are presented. The upper limits to the $\mathrm{Na}$ I interstellar line imply that the gas in the halo has a much larger value of the ratio of the column density of $\mathrm{Ca}$ II to that of $\mathrm{Na} \mathrm{I}$ than does the plane, and that this ratio is larger than the intermediate value obtained previously from a group of brighter halo stars. From this we deduce that there is gas up to at least $1 \mathrm{kpc}$ above the plane, and that this gas has much more Ca II relative to $\mathrm{Na} I$ than does the plane. The interstellar equivalent width corresponding to looking out of the Galaxy from the plane at $b^{\mathrm{II}}=90^{\circ}$ is about $300 \mathrm{~m} \AA$ for the stronger of the Ca II lines. The velocity measurements imply that this gas is moving slowly toward the plane in a time scale such that replenishment of the halo gas is necessary.

Subject headings: abundances — faint blue stars — galactic structure — interstellar matter
\end{abstract}

\section{INTRODUCTION}

A survey of faint blue stars near the galactic poles was compiled by Feige (1958). This list provides a useful probe for investigating the interstellar medium (IM) far above the plane. The principal problem of the Feige stars is that their luminosities are not known accurately. Some of the stars are subluminous or field horizontal-branch stars, and at the low dispersions available the luminosity of the stars and hence the heights above the plane $(Z)$ are uncertain. Previous studies by Greenstein (1968) and Sargent and Searle (1968) have focused on the spectra of the stars themselves and of the interstellar K-line of Ca II. New observations of the interstellar $\mathrm{Ca}$ II and $\mathrm{Na}$ I lines in the Feige stars are presented in $\S$ II. The interpretation of these observations in terms of the peculiar relative abundances of $\mathrm{Ca} \mathrm{II}, \mathrm{NaI}$, and $\mathrm{HI}$, and also of the peculiar velocity distribution of the observed components, is discussed in $\S$ III. We find the Feige stars to be even more extreme, as compared with stars in the plane, than the brighter halo stars of Cohen (1974) in the peculiarities of the interstellar lines. We conclude from two separate lines of evidence that there must be gas containing $\mathrm{Ca}$ II at $Z=1 \mathrm{kpc}$, with a ratio of $\mathrm{Ca} I \mathrm{II} / \mathrm{Na}$ I much larger than in the plane, and an enhancement of $\mathrm{Ca}$ II relative to $\mathrm{H}$ I. In $\S$ IV we discuss a model where the halo gas is falling toward the plane and is continually being replenished, which model is forced upon us by the radial velocity data. We also speculate briefly on the relationship between the optical gas and the high-velocity radio gas.

\section{OBSERVATIONS}

A catalog of faint $\left(9.5<M_{V}<13.0\right)$ underluminous hot stars at high galactic latitudes was compiled by Feige (1958). Many of them are O and B stars which show interstellar lines. High-dispersion

* Operated by the Association of Universities for Research in Astronomy, Inc., under contract with the National Science Foundation. spectra of several of these objects taken at the Lick Observatory by R. Kraft, K. Anderson, W. L. W. Sargent, and L. Searle were generously loaned for this investigation. In addition, the plate files of the Hale Observatories were searched for additional spectra.

Thirty spectra of O- and B-type stars were taken in the blue on $103 \mathrm{a}-\mathrm{O}$ plates at Lick Observatory during the years 1965-1969 with a wavelength range between 3600 and $4600 \AA$ and a dispersion of $47 \AA \mathrm{mm}^{-1}$. Twenty-two of these were among the spectra used by Sargent and Searle (1968). Nine more were taken in the blue at Palomar Mountain in 1966-1967 using baked IIa-O plates. Their range was $3400-4800 \AA$ with a dispersion of $10 \AA \mathrm{mm}^{-1}$. Using 39 plates, representing 23 different stars, we measured equivalent widths and radial velocities from the $\mathrm{Ca}$ II $\mathrm{K}$-line at $3933 \AA$. Although all but eight of the 39 blue spectra had been previously measured by Sargent and Searle (1968) or Greenstein (1968), we felt it necessary for reasons of uniformity with other papers in this series to measure all the spectra ourselves. A comparison of our measurements with those previously published is given in columns (2) and (3) of Table 2.

In addition, seven spectra were taken at the Lick Observatory in 1970 on 103a-D plates using a Varo image intensifier (Zappala 1971) with a central wavelength of $6100 \AA$ and a dispersion of $35 \AA \mathrm{mm}^{-1}$. These provided upper limits on $\mathrm{Na} I$ abundances.

The 23 stars which were chosen for study are listed in Table 1. Their color excesses were calculated using observed $B-V$ given by Sargent and Searle (1968) and the calibrations of Schild, Peterson, and Oke (1971). Distances above the galactic plane were determined using absolute magnitudes from Allen (1963) and the apparent magnitudes listed by Sargent and Searle (1968), assuming that the stars are either main-sequence or horizontal-branch types. Distances are given in Table 1 only for stars with relatively normal spectra, so that more or less reliable values of $Z$ could be calculated. 
TABLE 1

Basic parameters of observed Feige stars

\begin{tabular}{|c|c|c|c|c|c|c|}
\hline No. & Spectral Type & $\mathrm{E}_{\mathrm{B}-\mathrm{V}}$ & $\mathrm{v}_{\text {LSR }}$ & $v_{L S R}^{i}$ & $\begin{array}{l}\text { No. of } \\
\text { Blue Spectra }\end{array}$ & $\mathrm{z}$ \\
\hline $\begin{array}{l}\text { F10 } \\
\text { F11 } \\
\text { F16 } \\
\text { F23 } \\
\text { F25 }\end{array}$ & $\begin{array}{l}\text { Ao } \\
\text { Bp } \\
\text { AO } \\
\text { B8, B6 } \\
\text { B7, B6 }\end{array}$ & $\begin{array}{l}0.0 \\
0.0 \\
0.09\end{array}$ & $\begin{array}{l}-19 \\
-46: \\
+\quad 9 \\
-27 \\
+36\end{array}$ & $\begin{array}{l}-16^{1} \\
-6 \\
+12^{1} \\
-15 \\
+7\end{array}$ & $\begin{array}{l}1 \\
2 \\
1 \\
1 \\
1\end{array}$ & $\begin{array}{l}1500 \\
2700\end{array}$ \\
\hline $\begin{array}{l}\text { F29 } \\
\text { F34 } \\
\text { F36 } \\
\text { F40 } \\
\text { F51 }\end{array}$ & $\begin{array}{l}\text { B4 } \\
\text { Bp, sdo } \\
\text { sdB } \\
\text { B4, B5 } \\
\text { B6, B8 }\end{array}$ & $\begin{array}{l}0.06 \\
0.0 \\
0.0 \\
0.03 \\
0.0\end{array}$ & $\begin{array}{l}-12 \\
+61: \\
+27 \\
+79 \\
+41\end{array}$ & $\begin{array}{l}-9 \\
-29: \\
-5 \\
-18\end{array}$ & $\begin{array}{l}3 \\
1 \\
1 \\
2 \\
2\end{array}$ & $\begin{array}{l}1800 \\
2900 \\
1400\end{array}$ \\
\hline $\begin{array}{l}\text { F56 } \\
\text { F65 } \\
\text { F66 } \\
\text { F70 } \\
\text { F71 }\end{array}$ & $\begin{array}{l}\text { B8, } \mathrm{B} 6 \\
\mathrm{~B} 2, \text { sdB } \\
0, \text { sdB } \\
\mathrm{B} 8, \mathrm{BW} \\
\mathrm{B} 9, \mathrm{~B} 8 \mathrm{III}\end{array}$ & $\begin{array}{l}0.01 \\
0.0 \\
0.0 \\
0.01 \\
0.0\end{array}$ & $\begin{array}{l}+43 \\
+70 \\
+\quad 6 \\
+\quad 26 \\
+\quad 3\end{array}$ & $\begin{array}{l}-4 \\
+67^{1} \\
+1 \\
-30^{1} \\
-11^{1}\end{array}$ & $\begin{array}{l}4 \\
2 \\
1 \\
1 \\
1\end{array}$ & 1100 \\
\hline $\begin{array}{l}\text { F80 } \\
\text { F84 } \\
\text { F86 } \\
\text { F92 } \\
\text { F99 }\end{array}$ & 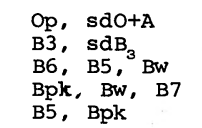 & $\begin{array}{l}0.04 \\
0.0 \\
0.02 \\
0.01\end{array}$ & $\begin{array}{l}+53 \\
+179 \\
-12 \\
+19\end{array}$ & $\begin{array}{l}+49^{1} \\
+31 \\
-8 \\
+8\end{array}$ & $\begin{array}{l}1 \\
1 \\
4 \\
4 \\
0\end{array}$ & \\
\hline $\begin{array}{l}\text { Flol } \\
\text { Fl10 } \\
\text { Flil }\end{array}$ & $\begin{array}{ll}\mathrm{Ao}, \mathrm{Bw} \\
\mathrm{Bp}, \text { sdo } \\
\mathrm{B} 8, \mathrm{Bw}\end{array}$ & $\begin{array}{l}0.0 \\
0.0 \\
0.11\end{array}$ & $\begin{array}{l}+56 \\
+33 \\
-31\end{array}$ & $\begin{array}{l}+53^{1} \\
-1^{1}\end{array}$ & $\begin{array}{l}1 \\
1 \\
1\end{array}$ & \\
\hline
\end{tabular}

${ }^{1} \lambda 3933 \AA$ probably or certainly stellar.

Radial velocities were measured for the stars and for the interstellar $\mathrm{Ca}$ II $\mathrm{K}$-line using the oscilloscope Grant machine of Kitt Peak National Observatory (KPNO) and are listed, relative to the local standard of rest, in Table 1. The stellar radial velocities are based on the lines $\mathrm{H} \gamma, \mathrm{H} \delta, \mathrm{H} 8$ to $\mathrm{H} 11$, plus the strong $\mathrm{He} \mathrm{I}$ lines when visible. In several cases where the lines are broad, the stellar $V_{r}$ are quite uncertain. Although there is only one interstellar line ( $\lambda 3933)$, it is sharp, and therefore we expect the $V_{r}^{i}$ velocities to be as accurate as $V_{r}$. The general accuracy is shown by the good agreement between the two measurements for F10 and F16, which are A0 stars, and by the good

TABLE 2

$\mathrm{w}_{\lambda}$ for interstellar lines in the Feige stars

\begin{tabular}{lcccc}
\hline \hline & $\mathrm{W}_{\lambda}$ & $\mathrm{W}_{\lambda}$ & $\mathrm{W}_{\lambda}$ & $\mathrm{w}_{\lambda}$ \\
Star & 3933 & $3933^{*}$ & 5889 & 5895 \\
\hline F11 & 110 & & & \\
F23 & $300:$ & 250 & & \\
F25 & 260 & 370 & & \\
F29 & 295 & 370 & 320 & 300 \\
F34 & $\leq 70$ & & & \\
F36 & $\leq 75$ & & & \\
F40 & 305 & 350 & $\leq 110$ & \\
F51 & 240 & 185 & $\leq 110$ & \\
F56 & 340 & 280 & $\leq 110$ & \\
F66 & $200:$ & 170 & & \\
F84 & $290:$ & 370 & & \\
F86 & 135 & 115 & $\leq 110$ & \\
F92 & 255 & 260 & $\leq 110$ & \\
F99 & & 155 & $\leq 110$ & \\
F110 & $\leq 100$ & & & \\
\hline
\end{tabular}

*mean of Sargent and Searle (1968) and Greenstein (1968) agreement with the measurements of Berger (1963) for the nine cases in common. We expect that in cases where there is only one spectrogram available, $V_{r}^{i}$ should be accurate to $\pm 15 \mathrm{~km} \mathrm{~s}^{-1}$.

The PDS digital microphotometer at KPNO was used to scan the spectra. Calibration exposures taken on either side of the stellar spectra allowed the conversion of density into intensity. Equivalent widths (Table 2) were measured for the $\mathrm{Ca}$ II $\mathrm{K}$-line and $\mathrm{Na}$ I $\mathrm{D}$ lines. The width of the K-lines, combined with the radial velocity data, provided a good discriminant between stellar and interstellar $\mathrm{K}$-lines in suspicious cases. The full widths at half-maximum for $\mathrm{Ca}$ II were typically on the order of $1-2 \AA$, and the interstellar lines were barely resolved. The $\mathrm{Ca}$ II $\mathrm{H}$-line is too blended with $\mathrm{H} \epsilon$ at such a low dispersion to be measured.

\section{DISCUSSION}

The pattern of $W_{\lambda}$ given in Table 2 for the interstellar lines in the Feige stars is rather different from that observed in the plane (see, for example, Cohen 1975). Normally, in the plane, the stronger $\mathrm{Ca}$ II line is slightly weaker than the stronger line of the yellow $\mathrm{Na}$ I doublet, except in a few cases with high radial velocities in the LSR system. Here there are seven measurements or limits for the ratio $\mathscr{W}=W_{\lambda}$ (Ca II $\lambda 3933) / W_{\lambda}$ (Na I $\left.\lambda 5889\right)$. In only one case is $\mathscr{W}$ less than 1. In four of the six cases with upper limits for $W_{\lambda}$ of the $\mathrm{Na}$ I line, $\mathscr{W}$ is greater than 2 . In the other two cases, the $\mathrm{Ca}$ II line is only slightly stronger than the Na I upper limit, so that all we can say is $\mathscr{W}>1$. If we assume that the doublet ratios are the same for the $\mathrm{Ca}$ II and $\mathrm{Na}$ I doublets, then $\mathscr{W}>2$ implies that $N(\mathrm{Ca}$ II $) / N(\mathrm{Na} \mathrm{I})>4$. Any reasonable 


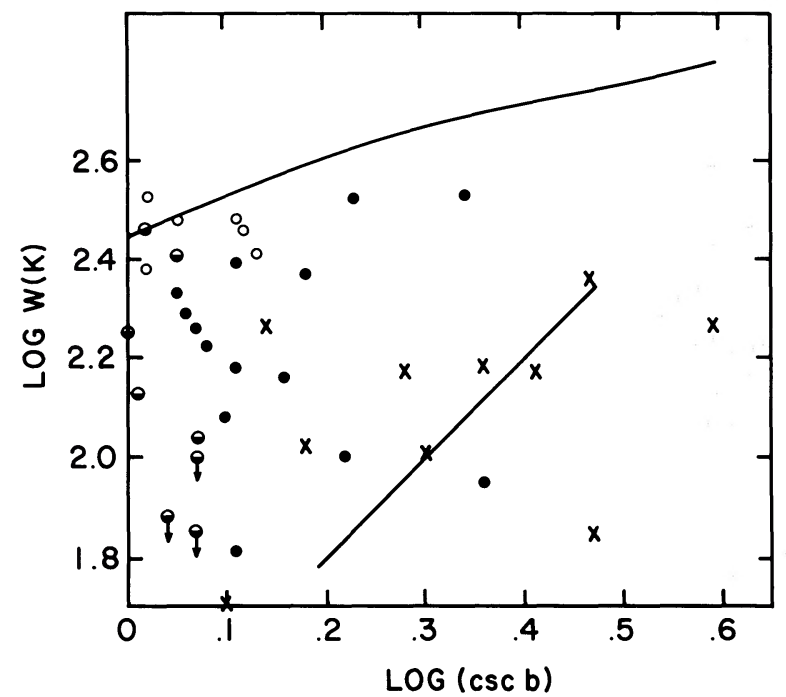

FIG. 1.-A plot of the equivalent width of the interstellar $\mathrm{K}$-line of $\mathrm{Ca}$ II as a function of $\csc \left(b^{\mathrm{II}}\right)$. Open circles, Feige stars not classified as subdwarfs or peculiar spectroscopically. Half-filled circles, Feige stars which are probably subluminous. Filled circles, the brighter halo stars (group B) which have $Z>500 \mathrm{pc}$, while the $\times$ 's are the halo stars with $Z<500$ pc. The lower solid line represents the theoretical prediction for unsaturated lines (eq. [1]), while the upper solid line is the predicted curve for saturated lines.

allowance for the different curve-of-growth effects expected for the D-lines, with $W_{\lambda} \leq 100 \mathrm{~m} \AA$ as compared to $W_{\lambda}=200$ to $300 \mathrm{~m} \AA$ for the stronger Ca II line, implies that for $\mathscr{W}>2, N(\mathrm{Ca}$ II $) / N(\mathrm{Na}$ I $)>8$. We therefore argue that the one case with $\mathscr{W}<1$ is that of a cloud very close to the plane on the line of sight to a distant halo star. The material actually far above the plane has $\mathscr{W}>2$; hence, $N(\mathrm{Ca}$ II $) / N(\mathrm{Na}$ I $)$ $>8$. If we compare this with the summary given in Table 6 of Cohen (1975), we see that the Feige stars represent a more extreme case of the characteristics ascribed to the brighter halo stars by Cohen (1974). This is not unreasonable, since we expect the Feige stars, with fainter apparent magnitudes than those given for the brighter halo stars by Cohen (1974), to have, on the average, higher values of $Z$. This strengthening of the halo pattern of interstellar lines (i.e., strong $\mathrm{Ca}$ II relative to $\mathrm{Na}$ I) in the Feige stars implies that there is actually material high in the halo between the mean $Z$ of the Feige stars and that of the brighter stars of Cohen (1974)-i.e., material at least up to $Z=1 \mathrm{kpc}$.

Is there any other evidence that there is actually material containing $\mathrm{Ca}$ II that high in the halo? In Figure 1 we plot the logarithm of $W_{\lambda}(K)$ versus the logarithm of $\csc \left(b^{\mathrm{II}}\right)$. The Feige stars are divided into those which appear to have normal spectra (open circles) and those which are classified by either Greenstein (1968) or Sargent and Searle (1968) as subdwarfs. We expect the latter group to be subluminous and hence closer to the plane. The dots indicate the stars of Cohen (1974) and Münch and Zirin (1961) with $Z>500 \mathrm{pc}$, which are in the mean not as high as the fainter Feige stars. (We shall refer to these brighter halo stars as group $B$.) The $\times$ 's indicate the stars of Cohen (1974) with $Z<500 \mathrm{pc}$, but $b^{\text {II }}>15^{\circ}$. If the interstellar medium above the plane is idealized as consisting of plane-parallel layers, then for unsaturated lines,

$$
W_{\lambda}(K)=W^{0} f\left(Z / Z_{\text {top }}\right) \csc \left(b^{\mathrm{II}}\right) .
$$

Here $W^{0}$ is the $W_{\lambda}$ resulting from looking at $b^{\text {II }}=90^{\circ}$ at a value of $Z$ above the layer of gas whose upper limit in height above the plane is $Z_{\text {top }}$. For layers of uniform density, $f\left(Z / Z_{\text {top }}\right)=Z / Z_{\text {top }}$, while in all cases $f\left(Z / Z_{\text {top }}\right)$ increases monotonically to 1 at $Z=Z_{\text {top }}$. The straight line on Figure 1 represents equation (1) in the regime of its validity, where $W_{\lambda}(K)$ is small. The curved line is an attempt to correct equation (1) for saturation in the case of large values of $W_{\lambda}(K)$.

Not surprisingly, the observations displayed in Figure 1 show the expected behavior. $W^{0}$ appears to be about $300 \mathrm{~m} \AA$ as judged by the Feige stars with normal spectra. (Greenstein [1968] obtained $W^{0}=$ $400 \mathrm{~m} \AA$ from his version of Figure 1 with the data then available.) Those Feige stars which from spectroscopic evidence seem to be subluminous indeed fall toward the lower part of the figure, implying a lower value of $Z$. The stars of group B appear to have heights intermediate between those of the subdwarf Feige and normal Feige stars. Most important, the value of $W_{\lambda}(K)$ at $b^{\mathrm{II}}$ close to 0 keeps increasing as the mean expected value of $Z$ increases; i.e., the normal Feige stars have values of $W_{\lambda}(K)$ larger than those of group $\mathrm{B}$ stars at similar galactic latitudes. This is conclusive evidence for gas at heights of at least $1 \mathrm{kpc}$.

Now let us consider the question of whether the halo gas has an excess of $\mathrm{Ca}$ II relative to $\mathrm{HI}$, or a deficit of $\mathrm{Na}$ I. Although the values of $E_{B-V}$ are uncertain, a maximum value is $0.10 \mathrm{mag}$, implying a maximum value of $\log N(\mathrm{H} \mathrm{I})=20.8 \mathrm{~cm}^{-2}$, using the ratio $E_{B-V} / N\left(\mathrm{H}_{\mathrm{I}}\right)$ given by Jenkins and Savage (1974). In Cohen (1974) we demonstrated that the ratio $E_{B-V} / N(\mathrm{H} \mathrm{I})$ is not smaller at high galactic latitudes than it is in the plane. From the values of $W_{\lambda}$ for the cases with $\mathscr{W}>2$, we obtain a minimum of $\log$ $N($ Ca II $)=12.8 \mathrm{~cm}^{-2}$ (since we do not know the doublet ratio), and a maximum value of $\log N(\mathrm{Na} \mathrm{I})=$ $11.8 \mathrm{~cm}^{-2}$ (since we have only an upper limit on $W_{\lambda}$ ). The resulting ratios of minimum $\log [N(\mathrm{Ca} \mathrm{II} / \mathrm{H} \mathrm{I})]=$ -8.0 indicate clearly, when compared with Table 6 of Cohen (1975), that there is a strong excess of Ca II relative to $\mathrm{H}$ I as compared with the plane. This again is an amplification of the behavior of the brighter halo stars discussed by Cohen (1974). Of course, it may be that most of the $\mathrm{H} \mathrm{I}$ is in the plane, and the hydrogen above the plane, if present, is ionized, whereas most of the Ca II cannot be in the plane.

Let us now consider the measured radial velocities in the LSR system. First, the normal B stars located at $Z>500$ pc must be runaway B stars, and should have large stellar radial velocities. This is certainly the case for F40, F51, and F56, which strengthens the evidence from their spectral types that these stars are far above the plane. 


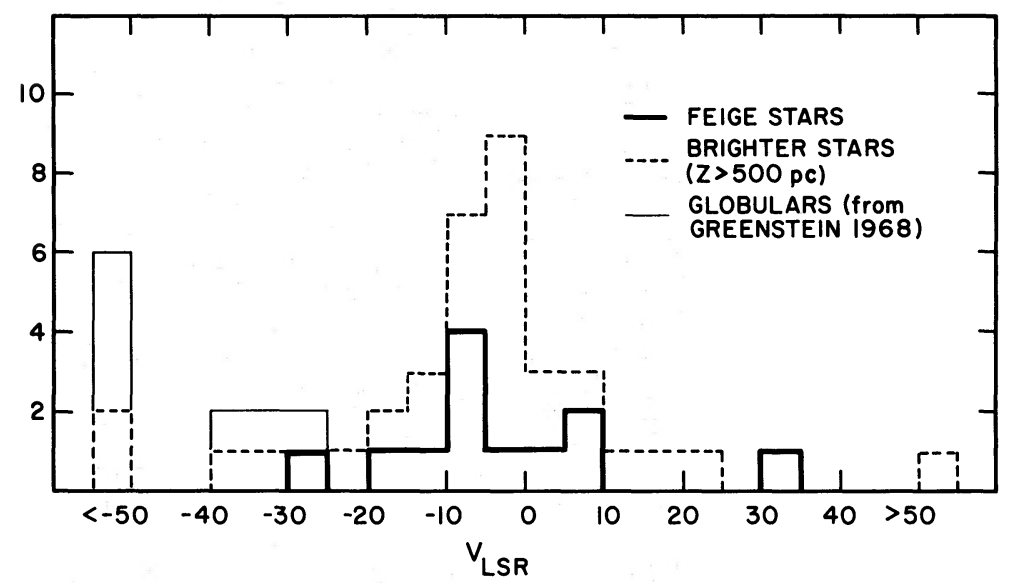

FIG. 2.-A histogram of the velocities in the LSR system of the components of the interstellar lines observed in various groups of halo stars. The heavy solid line refers to the Feige star velocities; the dotted line, to the stars of group B (the brighter halo stars); the thin solid line, to the globular cluster stars.

We also note that the agreement between the predicted $V_{\mathrm{LSR}}$ from galactic rotation,

$$
V_{\mathrm{LSR}}^{\text {pred }}=A r \sin \left(2 l^{\mathrm{II}}\right) \cos ^{2} b^{\mathrm{II}},
$$

and the observed $V_{\mathrm{LSR}}$ is terrible, and in most cases no value of $r$ can be deduced which is less than the distance of the star. Although the errors in the measurement of $V_{r}$ are large as a result of the low-dispersion spectra, stronger evidence for the lack of corotation with the plane for gas at high galactic latitudes has been discussed, using more accurate radial velocities by Cohen (1974).

In Figure 2 we plot a histogram of $V_{\mathrm{LSR}}$ for each interstellar component for several groups of stars: the Feige stars from the data of Table 1, the brighter halo stars of group B, and the measurements of Greenstein (1968) for globular cluster stars. In the last group, there may be some problem of contamination by faint background stars in the cluster, and hence these velocities are the least reliable. The distribution appears skewed toward negative values of $V_{\mathrm{LSR}}$. This impression is confirmed by the averages given in Table 3. The last entry was an attempt to remove the effect of the local components arising within the plane $(Z<100 \mathrm{pc})$, which will have $V_{\mathrm{LSR}}$ close to 0 . We note that all the averages are negative, suggesting that the gas in the halo that is seen in the Ca II interstellar line on the average is falling toward the plane at a radial velocity $\left(V_{\mathrm{LSR}}\right)$ of about $-6 \mathrm{~km} \mathrm{~s}^{-1}$. This is much smaller than the velocities of about $-100 \mathrm{~km} \mathrm{~s}^{-1}$

\section{TABLE 3}

Average Value of $V_{\text {LSR }}$

\begin{tabular}{cccc}
\hline \hline Parameter & Feige Stars & Group B & $\begin{array}{c}\text { Feige }+ \text { Group B } \\
|V| \geq 5 \mathrm{~km} \mathrm{~s}^{-1}\end{array}$ \\
\hline $\bar{V}_{\text {LSR }} \ldots \ldots \ldots \ldots$ & -4 & -6 & -7.5 \\
$\begin{array}{r}\text { Number of } \\
\text { components } \ldots\end{array}$ & 12 & 26 & 30 \\
\hline
\end{tabular}

(Dieter 1971) ascribed to the high-velocity clouds of neutral hydrogen falling toward the disk.

The interpretation of the halo gas, derived from the optical interstellar lines, as slowly moving toward the plane, is similar in some respects to that of the very early radio studies as analyzed by Helfer (1962) and Blaauw (1962). It is not clear whether the negative velocities are confined largely to the region $l^{\mathrm{II}}$ from $100^{\circ}$ to $180^{\circ}$ as suggested by Blaauw. Most of Heiles gas $\left(-20 \leq V_{\mathrm{LSR}} \leq-92 \mathrm{~km} \mathrm{~s}^{-1}\right)$ is in that region. Furthermore, the most extreme negative velocity interstellar components in stars are at galactic longitude $84^{\circ}$ and $183^{\circ}$, with almost no components observed at longitudes between $85^{\circ}$ and $180^{\circ}$. If all the interstellar components in the three stars near $l^{\mathrm{II}}=84^{\circ}$ and $184^{\circ}$ with the largest negative velocities are removed from the sample, the average LSR velocity of the interstellar components in the remaining halo stars (excluding globular cluster stars) becomes almost 0 . Thus it is not clear whether the predominance of negative radial velocities is global or is confined to a range in galactic longitude from about $80^{\circ}$ to $185^{\circ}$.

The most peculiar $V_{\mathrm{LSR}}$ in Figure 1 is that of a component of HD $203664 \mathrm{at}+76 \mathrm{~km} \mathrm{~s}^{-1}$. This star is not in the direction of the region of the Magellanic stream with large positive radial velocities (Mathewson, Cleary, and Murray 1974), and therefore the origin of this component is puzzling.

\section{CONCLUSIONS}

From the interstellar lines observed in the Feige stars, we have found that there must be gas at least $1 \mathrm{kpc}$ above the plane. This gas has a ratio of $N(\mathrm{Ca} \mathrm{II}) /$ $N(\mathrm{Na}$ I) at least 8 times larger than that observed for the plane. Furthermore, the $N(\mathrm{Ca} \mathrm{II}) / N(\mathrm{H} \mathrm{I})$ ratio is larger than that in the plane. We have also noted that the distribution of radial velocities in the LSR system is skewed negative. This holds at both positive and negative values of galactic latitude. We thus can imagine a model where the halo gas is, on the average, 
falling toward the plane with a mean $V_{\mathrm{LSR}}$ of $5 \mathrm{~km}$ $\mathrm{s}^{-1}$. Material $1 \mathrm{kpc}$ above the plane will require no more than $2 \times 10^{8}$ years to reach the plane, and the fall time could be shorter due to gravitational acceleration near the plane. This implies that the halo is being emptied of gas either globally or in a certain range of galactic longitude, and hence must be replenished either by gas ejected from the plane (perhaps from supernova explosions), by mass loss from stars in the halo, or by intergalactic gas falling in (Oort 1970). The mass of the halo gas inferred from the observed interstellar lines is $1-6 \times 10^{7} M_{\odot}$ (Cohen 1975). If the mass loss from halo to plane is global, this mass loss is larger than any reasonable estimate of the mass ejection expected in the course of stellar evolution for stars in globular clusters, which is of the order of $10^{5} M_{\odot}$ of gas in $10^{8}$ years (Knapp, Rose, and Kerr 1974). The extremely uncertain estimate of mass loss by Mira variables with $Z>1 \mathrm{kpc}$ in the halo is
$10^{-1} M_{\odot}$ per year (Gehrz and Woolf 1971). This may be sufficient to account for the gas seen optically, although it would be hard to explain the presence of clouds with positive radial velocities. If this is the source of most of the halo gas, one might expect interstellar grains in the halo.

The relationship between the radio high-velocity gas and the gas seen optically remains very unclear. Observations of interstellar lines in spectra of external galaxies and globular cluster stars as well as a search for halo stars with $l^{\text {II }}$ between $90^{\circ}$ and $180^{\circ}$ would help a great deal in elucidating the location and motion of the gas.

We are extremely grateful to Drs. R. P. Kraft, K. Anderson, J. L. Greenstein, and W. Sargent for providing the spectra on which this investigation is based.

\section{REFERENCES}

Allen, C. W. 1963, Astrophysical Quantities (2d ed.; London: Athlone).

Berger, J. 1963, Pub. A.S.P., 75, 393.

Blaauw, A. 1962, in The Distribution and Motion of Interstellar Matter in Galaxies, ed. L. Woltjer (New York: W. A. Benjamin), p. 48.

Cohen, J. G. 1974, Ap. J., 194, 37.

-_—. 1975, ibid., 197, 117.

Dieter, N. 1971, Astr. and Ap., 12, 59.

Feige, J. 1958, $A p$. J., 128, 267.

Gehrz, R. D., and Woolf, N. J. 1971, Ap. J., 165, 285.

Greenstein, J. L. 1968, Ap. J., 152, 431.

Heiles, C., and Habing, H. J. 1974, Astr. and Ap. Suppl., 14, 1.

Helfer, H. L. 1962, in The Distribution and Motion of Interstellar Matter in Galaxies, ed. L. Woltjer (New York: W. A. Benjamin), p. 26.
Jenkins, E. B., and Savage, B. D. 1974, Ap. J., 187, 243.

Knapp, G. R., Rose, W. K., and Kerr, F. J. 1974, preprint.

Mathewson, D. S., Cleary, M. N., and Murray, J. D. 1974, Ap. J., 190, 291.

Münch, G., and Zirin, M. 1961, Ap. J., 133, 11.

Oort, J. M. 1970, Astr. and Ap., 1, 390.

Sargent, W. L., and Searle, L. 1968, Ap. J., 152, 443.

Schild, R. G., Peterson, D. M., and Oke, J. B. 1971, Ap. J., 166, 95.

Zappala, R. R. 1971, Proc. Conference on Late-Type Stars, ed. G. W. Lockwood and H. M. Dyck (Tucson: Kitt Peak National Observatory), p. 1.

Judith G. Cohen: Kitt Peak National Observatory, P.O. Box 26732, Tucson, AZ 85726

Debra Meloy: Princeton University Observatory, Princeton, NJ 08540 
